\title{
LA-5110
}

EXOTIC: A Computer Code for Energy Levels of Exotic Atoms 
This report was prepared as an account of work sponsored by the United States Government. Neither the Unired States nor the United States Atomic Energy Commission, nor any of their employees, nor any of their contractors, subcontractors, or their employees, makes any warranty, express or implied, or assumes any legai liability or responsibility for the accuracy, completeness or usefulness of any information, apparatus, product or process disclosed, or represents that its use would not infringe privately owned rights.

Printed in the United States of America. A.vailable from National Technical Information Service

U. S. Department of Commerce 5285 Port Royal Road Springfield, Virginia 22151

Price: Printed Copy $\$ 3.00$; Microfiche $\$ 0.95$ 
LA-5110

UC-34

ISSUED: January 1973

\section{EXOTIC: A Computer Code for Energy Levels of Exotic Atoms}

\author{
by \\ Justus H. Koch" \\ Morton M. Sternheim**
}

This report whs preperred

aponsored by the prepared as an account of wo the United States United States Government. Neith Commission. their contractors, any of their employees, nor any their contractors, subcontractors, or their employe mikes any warranty, express or implied, or assumes a legal lis bility or responsibility for the accuracy, cor pleteness or usefulness of any information, appirat product or process disclosed, or represents that its would not infringe privately owned rights.

\footnotetext{
"Stanford University, California

* University of Massachusetts, Amherst, Massachusetts
}

This work was supported partially by the National Science Foundation. 


\section{ABSTRACT}

EXOTIC is a FORTRAN code which finds the elgenfunctions and complex elgenvalues of the Klein-Gordon equation for $\mathrm{K}$-mesic atoms. Provisions are marie to allow for various models of the nuclear interaction. With some modifications this program can also be used for other exotic atoms.

\section{INTRODUCTION}

EXOTIC Is a FORTRAN code which finds the eigenfurctions and coiplex elgenvalues of the RleinGordon equation for K-mesic atoms. It contains an optical potential, $\mathrm{V}_{\mathrm{N}}$, which is proportional to the nuclear density, and a finite charge distribution Coulomb potential, $v_{C}$ : $\left[-\frac{d^{2}}{d r^{2}}+\frac{\ell(\ell+1)}{r^{2}}-\left(E-v_{C}\right)^{2}+m^{2}+2 E V_{N}\right] u=0$.

Here $\mathrm{m}$ ts the kaon reduced mass.

This program can also be used for other exotic atoms. With some modification of $v_{N}$, the KrellEricson local form ${ }^{1}$ of the Klsslinger potential ${ }^{2}$ for plons could be incorporated. If one neglects fine structure effects, it can also be used for $\vec{p}$ and $\Sigma^{-}$atoms.

The nuclear potential, $V_{N}$, can be chosen to be of the form

$$
V_{N}(r)=-(2 \pi / m)\left(1+m / m_{N}\right) b p(r)
$$

for

$$
\rho_{n}(r) / N=\rho_{p}(r) / Z=\rho(r) / A,
$$

or

$v_{N}(r)=-(2 \pi / m)\left(1+m / m_{N}\right)\left[b_{n} \rho_{n}(r)+b_{p} \rho_{p}(r)\right]$
Here $m_{N}$ is the proton mass and the nuclear densities are normalfzed to

$$
\int \rho_{p} d^{3} r=z, \int \rho_{n} d^{3} r=N \text {, and } \int \rho d^{3} r=A \text {. }
$$

ithe complex b's are the "effective scattering lengths" for the $K-N$ systems.

The shifts $\varepsilon$ and widths $\Gamma$ resulting from the strong interaction are defined according to

$$
E-1 \Gamma / 2=E(\text { calculated })-E(\text { point Coulomb }),(3)
$$

i.e., $\varepsilon>0$ corresponds to a repulsive shift. Note that Eq. (3) will also include the small effents due to a finite charge distribution if $v_{C}$ does not correspond to a point charge. EXOTIC has been used to calculate energy levels of several $\mathrm{K}$-mesic atoms. 3,4

\section{NUMERICAL METHODS}

The differential equation, Eq. (1), is integrated using standard techniques. ${ }^{4}$ A modified Noumerov 5 method is used to integrate out from the origin and in from a radius, $r_{n}$, where the wave function is closely approximated by its asymptotic farm. An Iteration procedure discussed below reduces the discontinufty in $u^{\prime} / u$ at the matching point as the eigenvalue is approached. 


\section{A. Integration}

To begin the outward integration for \& state with quantum numbers $n$, $\ell$, we use

$u(0)=0, u\left(r_{1}\right)=r_{1}^{\ell+1}$.

To begin the Inward integration, we use

$$
\begin{aligned}
& u\left(r_{n}\right)=\text { const. } \\
& u\left(r_{n-1}\right)=u\left(r_{n}\right) e^{-1 k\left(r_{n}-r_{n-1}\right)}
\end{aligned}
$$

where $k$, the average wave number in the starting region, is

$$
\begin{aligned}
k & =\frac{1}{2}\left\{\left[E_{0}-v_{C}\left(r_{n}\right)\right]^{2}-m^{2}\right\}^{\frac{1}{2}} \\
& +\frac{1}{2}\left\{\left[E_{0}-v_{c}\left(r_{n-1}\right)\right]^{2}-m^{2}\right\}^{\frac{1}{2}}, \text { Im } k<0 .
\end{aligned}
$$

The Initial trial elgenvalue, $E_{0}$, is elther the point Coulomb value or one inferred from preceding calculations with slightly different optical potentials.

Except for s-states, the wave function has two classical turning points. The Inward integration is started where $u\left(r_{n}\right)$ is a factor $e^{-15}$ times its value at the outer turning point. (An optional input control permits this factor to be changed.) Normally, we take $r_{m}$, the point wherc we match the two wave functions, to be the inner turaing point. (This can also be adjusted with an optional input.)

The numbers of mesh points in the two integrations are chosen independently. This allows the use of a finer mesh in the cutward integration, thus producing a greater accuracy in calculating the nuclear shifts for a given total number of points.

\section{B. Iteration Procedure}

Blatt ${ }^{6}$ has given a very efficient method of determining the correct efgenvalue from a reasonably good trial eigenvalue based on the variational principle. Let $\mathrm{E}_{0}$ be the trial eigenvalue, and $\mathrm{u}_{1}^{0}$ and $u_{2}^{o}$ be the corresponding solutions of Eq. (1) for $r\left\langle r_{m}\right.$ and $r>r_{m}$, respectively. The normalization of $u_{2}^{o}$ is adfusted so that $u_{1}^{0}\left(r_{m}\right)=u_{2}^{0}\left(r_{m}\right)$. Define $\mathrm{E}_{1}$ by

$$
E_{1}^{2}=N^{-1} \int u^{o *}\left[-\frac{d^{2}}{d r^{2}}+G(r)\right] u^{o} d r,
$$

where

$$
\mathrm{N}=\int \mathrm{u}^{\mathrm{o}} \mathrm{u}^{0} \mathrm{dr}
$$

and

$$
G(r)=\ell(\ell+1) / r^{2}+2 E_{O} V_{C}-V_{C}^{2}+2 E_{O} V_{N}+m^{2}
$$

$E_{1}$ is an improved trial eigenvalue. Since $u^{0}$ satisfles

$$
\left[-\frac{d^{2}}{d r^{2}}+G(r)\right] u^{o}(r)=E_{o}^{2} u^{o}(r),
$$

except at $\mathbf{r}=\mathbf{r}_{\mathrm{m}}$, we obtain

$$
E_{1}^{2}=E_{0}^{2}+\Delta \text {, }
$$

where $\Delta$ is a teri: arising from the discontinuity in $u_{0}^{\prime}$. Explicitly,

$$
\Delta=\left(u_{1}^{o *} u_{1}^{o}-u_{2}^{o *} u_{2}^{o}\right) / N
$$

Usual1y, we have found that this procedure converges extremely rapidly. If $E_{o}$ is not a sufficlently good starting value for this method to converge, the code will automatically find a better trial value, $E_{1}$, with a gradient search and eventually return to the Blatt 1 teration method.

\section{Accuracy and Speed}

Using 500 mesh points in each region, $1 . e .$, $\operatorname{NPTS}(1)=\operatorname{NPTS}(2)=500$, the maximura provided for in EXOTIC, we have reproduced the point Coulomb binding energies to a part in $10^{9}$, e.g., to $10^{-3} \mathrm{eV}$ or better for sulfur. Normally, with the optlcal potential, $V_{N}$, curned on, the eigenvalue iteration procedure requires on $1 y 1$ to 4 iterations to yield a binding energy stable to a part in $10^{8}$ or $10^{9}$, i.e., a shift and width stable to better than $1 \mathrm{eV}$. W1th $\operatorname{NPTS}(1)=\operatorname{NPTS}(2)=250$, a sequence of eigenvalue calculations for closely spaced complex well depth parameter values can be done at the rate of 5 per second on a CDC 6600 .

As noted above, various optional inputs are available. They make 1 possible to change the mesh sizes, the matching point, the starting point for the Inward integration, and the convergence criterion. Thus, one can determine empirically the 
optimum choices for these parameters and verify the accuracy for particular situations.

\section{ORGANIZATION OF PROGRAM}

The program 18 organized in the conventional way with a main program and several subroutines. Each of these will be discussed briefly below.

\section{A. EXOTIC (Main program)}

Th1s reads the input data, makes suitable enit conversions, calls the major subroutines, and prints the inputs and some intermediate quantities.

\section{B. WELLS}

Called by ExoTIC. At start of run it calls the subroutines which tabulate VR, VI, and VC, the real, imaginary, and Coulomb well shapes, respectively. For each set of (Jieh, Imb) values, it multiplies VR and VI by the appropriate factor.

C. WREAL, WRAC, WCOUL

Called by WELLS. These tabulate VR, VI, and VC for uniform spheres or Saxon-Woods densities.

D. EIGEN

Called by BXOTIC. Controls integration and iteration procedures, prints principal results.

E. SMOOTH, SMOOTH4

Called by EIGEN. Extrapolate results of preceding 3 or 4 calculations to provide starting $E_{0}$ for current value of $b$.

F. INT

Called by EIGEN. Integrates differential equation and obtains new trial eigenvalue $E_{1}$ using iteration procedure. (See Sec. II above.)

\section{G. FARRAY}

Called by INT. Tabulates F, where

$$
F=d^{2}\left[m^{2}-E^{2}+2 E\left(v_{C}+V_{N}\right)\right]+F 3,
$$

and

$$
F 3=d^{2}\left[L(L+1) / R^{2}-v_{C}^{2}\right]
$$

Here d is the step size, which is different in the two reglons.

\section{H. GRADIN}

Called by EIGEN. Used to obtain an $E$ only if Iteration procedure of INT falls to converge. I. PRINT

Called by EIGEN. Prints wave functions.
J. PERT

Called by EXOTIC. Calculates strong Interaction shifts at widths in first order perturbation theory using Klein-Gordon Coulomb wave functions. K. CHANGE

Called by EXOTIC. Changes specifled parameters and restarts same sertes of calculations. Ayoids need to read complete new input deck.

\section{PROGRAM INPUT}

All integer inputs must be right justified.

A. Basic Input Deck

Card 1: Title Card

Col. 2: A "1" Indicates the start of a new problem.

A "2" Indicates the end of the joh. Negat Ive entries in Col. 1-2 activate the change card procedure. (See Sec. IV.C below.)

Co1. 3-80: Title-to be printed as output heading.

Card 2: Control Card

tCol. 1-10*: 2 -nuclear charge number.

tCol. 11-20: ANUM - nuclear mass number. Neea not be rounded to an integer. (If blank or zero, $2 \mathrm{Z}$ is used.)

tCol. 21-30: PMASS - meson mass in MeV. (If blank or zero, the $\mathrm{K}^{-}$mass 493.84 MeV is used.)

Co1. 35*: N - principal quantum number of atomic state.

Co1. 40*: ' L - angular momentum quantum number of atomic state.

Col. 43-45: NPTS(1) - number of mesh points in inner region, $\leq 500$. (If $<50,250$ is used.)

Col. 48-50: NFTS(2) - number of mesh points in outer region, $\leq 500$. (If $<50,250$ is used.)

Co1. 55: IEXP - efgenvalue search or iteration procedure stops when fractional change in the energy-eigenvalue $<10^{- \text {IEXP }}$. (If blank or zero, 6 is used.)

fFloating point quantity; must have a decimal point. *This input must always be specifled. 
Co1. 58-60: IPR - potentlal array print control. IPR $=+n$ : Causes arrays to be printed at every nth point for each eigenvalue.

IPR $=-n:$ Causes arrays to be printed at every nth point for fj.rst eigenvalue only.

$|n| \leq 50$.

Arrays printed (in notation of Section III):

$F 1=2 d^{2} v_{C}$

$F 2=2 d^{2}\left(V_{C}+V_{N}\right)$

VR, real potential normalized to

ANUM.

VI, imaginary.

F, F3. See Eqs. (10) and (11).

Co1. 65 :

Co1. 70:

Col. 73-75:

Co1. 80:
INNER - if equal to 1 or 2 this will cause an extra card, number 6 , to be read. See below.

IQ - iteration print control.

IQ $=1:$ All trial eigenvalues and the corresponding discontinuity in the logarithmic derivative will be printed.

IQ $\neq 1$ : Only the first and last

step will be printed.

In a11 cases, the output con-

tains: input data, point coulomb

energy, Bohr radius, inner and outer turning points (RTURN), maximum radil of the Inner and outer integration (RMAX), two mesh sizes (DR), "effective scattering length," calculated shift and width, and complex wave number $k=\left(E^{2}-m^{2}\right)^{\frac{1}{2}}$.

IW - wave function print control. $I W=+n:$ Real, imaginary parts for every nth point.

$\mathrm{IW}=-\mathrm{n}$ : Magnitude, $\mathrm{i}$ hase.

$|n| \leq 50$.

IPT $=0$ or blank: Calculates exact eigenvalues and prints them.

IPT $=-1$ : Same as above, but also writes nuclear charge $Z$, shift EPS and width GAM on tape 93 (FORMAT

1X, F5.1, 2E15.6).

IPT $=+1$ : Calculates nuclear shift and width in perturbation theory.
IPT $=+2:$ As above, but also writes perturbation theory shifts and widths on tape 92; same FORMAT as for IPT $=-1$.

Card 3: Coulomb Card

Ali radil on cards 3,4 , and 5 are in fermis.

Co1. 10*: IC - Coulomb well. type.

1 - uniform sphere charge distrihution.

2 - Saxon-Wood:

$\rho=\rho_{0}(1+\exp [(R-R C) / A C])^{-1}$

tCol. 11-20*: $\mathrm{RC}$ - Coulomb radius.

If $\mathrm{IC}=1$, $\mathrm{RC}=$ radius of sphere.

If $\mathrm{IC}=2$, $\mathrm{RC}=$ half density radius.

†Col. 21-30: AC - thickness parameter, required for $I C=2$.

+Co1. 31-40: XN(1). Controls location of matchIng inner and outer wave functions. Matching is at the inner classical turning point $+\mathrm{XN}(1) / \operatorname{Im}\left(2 \mathrm{~mW}_{\mathrm{o}}\right)^{\frac{1}{2}}$, where $W_{0}$ is the point Coulomb energy, and $m$ is the meson mess, both in $\mathrm{f}^{-1}$. Should norma11y be left iiank or zero.

tCo1. 4i-50: XN(2). Controls location where outer wave function integration begins. This is $a$ : the outer classical turning point $+\mathrm{xN}(2) / \operatorname{Im}\left(2 \mathrm{~mW}_{0}\right)^{\frac{1}{2}}$. If blank or zero, 15 is used.

Should normally be left blank or

zero.

†Co1. 51-60: RTYPE - type of radial parameters. 0.0 - all radii are used as given. 1.0 - RC, RRE, RIM (see below) multiplied by (ANUM) ${ }^{1 / 3}$.

†Co1. 61-70: STEPO - fractionai step size for start of gradlent search. (If blank or $0,0.01$ is used. Should normally be left b1ank.)

Co1. 71-80: $\quad$ DENS $=1$ : Will cause the code to use a two-density potent1al, Eq. (2b). The format of cards $4^{\prime}$ and $5^{\prime}$, to be used in this case in place of cards 4 and 5 , is given below in Sec. IV.B.

fFloating point quantity; must have a decinal point. *This input must always be specified. 
Card 4: Real Well Card

Co1. 10: IR - real well type.

-1 - same well type and parameters

as Coulomb.

0 - no real we11.

1 - uniform sphere.

2 - Saxon-Wood.

tCo1. 11-20: RRE - real well radius.

Required for $I R=1,2$.

If $I R=1, R R E=$ sadlus of sphere.

If $\mathrm{IR}=2$, $R R E=$ half density radius.

tCo1. 21-30: ARE - rcal well thickness parameter, required for $\mathrm{IR}=2$.

tCo1. 31-40: BRO - initial value of real part of scattering length (in fermis).

tCo1. 41-50: DBR - increments in real part of scattering length (in fermis).

Co1. 51-60: NBR - number of real well depth values to be computed.

\section{Card 5: Imaginary Well Card}

Co1. 10:

I1 - Linaglnary well type.

-1 - same well type and parameters

as real well.

0 - no imaginary wel1.

1 - unfiorm spheiz.

2 - Saxon-Wood.

tCo1. 11-20: RIM - imaginary well radius.

Required for $I I=1,2$.

If $I I=1, R I M=$ radius of sphere.

If $I I=2$, RIM $=$ half density radius.

tCo1. 21-30: AIM - 1maginary well thlckness parameter, required for $I I=2$.

tCo1. 31-40: BIO - Inttial value of Imaginary part of scattering length.

tCo1. 41-50: DBI - Increments in imaginary part of scattering length.

Co1. 51-60: NBI - number of imagtnary we11 depth values to be computed.

Card 6: Startirig Values (Required only if INNER = 1 or 2 on card 2)

If INNER $=1$ :

tCo1. 1-10: WRL - starting value for real part of energy for eigenvalue search (in $\mathbf{f}^{-1}$ ).

tCo1. 11-20: WIG - starting value of 1mag1nary part of energy (in $\mathrm{f}^{-1}$ ). If 1 NNER $=2$ : The program prints $\chi^{2}$, experimental values for shift and width (Iacluding the errors) and puts the $\chi^{2}$ (CHISQ) on tape 91 [FORMAT (E15.6)]. The user can then in a separate run use the information stored on that tape to construct $\chi^{2}$

- maps, etc.

tco1. 1-10: Experimental shift (in keV).

tCo1. 11-20: Experimental error in shift (in keV),

tCo1. 21-30: Exper lmental width (in keV).

tCol. 31-40: Experimental error in width (in kev) B. Two Densities, $\rho_{n}$ and $\rho_{p}$

If DENS a 1 on card 3, the program will use separate denetties for neutrons and protons. There are no provisions made to execute b0-loops for the effective scattering lengths in this optional mode. Caró 4':

Col. 1-10: Well type, proton. Code for different types as above for card 4.

tCol. 11-20: Well radius for protons.

tCol. 21-30: Thickness parameter for proton well.

†Co1. 31-40: $\operatorname{Re} b_{p}$, see Eq. (2b).

tCo1. 41-50: Im $b_{p}$, see Eq. (2b).

tCol. 51-60: Leave blank.

Card 5': Same as card 4', but for neutrons. c. Change Cards

It is possible to change parameters between problems without making a complete new Input deck by using the procedure below.

Card 7:

Co1. 1-2: $\quad-J$, where $J$ is the number of parameters to be changed $(J \leq 8)$.

Col. 3-80: Title, to be printed as a headirg for the output.

Card 8:

Col. 1-2: 1st parameter number (see Table I).

tCol. 3-10: lst parameter value.

Col. 11-12: 2nd parameter number.

tCol. 13-20: 2nd parameter value.

etc.: With up to 8 parameter numbers and values on the card.

tFloating point quantity; must have a decimal point. *Th1s input must always be spectfled. 
TABLE I

\begin{tabular}{|c|c|}
\hline $\begin{array}{c}\begin{array}{c}\text { Parameter } \\
\text { Name }\end{array} \\
\end{array}$ & $\begin{array}{c}\text { Parameter } \\
\text { Number }\end{array}$ \\
\hline$z$ & 1 \\
\hline ANUM & 2 \\
\hline PMASS & 3 \\
\hline $\mathrm{RC}^{\mathrm{a}}$ & 4 \\
\hline$A C$ & 5 \\
\hline $\operatorname{RRE}^{a, b}$ & 6 \\
\hline ARE & 7 \\
\hline $\operatorname{RIM}^{a, c}$ & 8 \\
\hline$A T M$ & 9 \\
\hline $\mathrm{XN}(1)$ & 12 \\
\hline $\mathrm{XN}(2)$ & 13 \\
\hline $\mathrm{N}$ & 14 \\
\hline L & 15 \\
\hline $\operatorname{NPTS}(1)$ & 16 \\
\hline NPTS(2) & 17 \\
\hline
\end{tabular}

${ }_{\text {If }}^{\text {RTYPE }=1 \text {, ANUM }}{ }^{1 / 3}$ scaling will be used.

${ }^{b}$ If $I R=-1$, RRE w111 be set equal to RC, and ARE to $\mathrm{AC}$.

${ }^{c}$ If II $=-1$, RIM will be set equa I to RRE, and $A I M$ to ARE.

\section{REFERENCES}

1. M. Krell and T. E. O. Ericson," Energy Levels and Wave Functions of Pionic Atoms," Nucl. Phys. Bl1, 521 550 (1969).

2. L. S. Kisslinger, "Scattering of Mesons by Light Nuclei," Phys. Rev. 98, 761-76,5 (1955).

3. J. H. Koch and M. M. Sternhelm, "K--Nucleus Interaction and Its Interpretation," Phys. Rev. Letters 28, 1061-1063 (1972).

4. J. H. Koch, "Effects of Strongly Absorptive Interactions," Los Alamos Scientific Laboratory report LA-4980-T (November 1972).

5. K. Kunz, Nemerical Analysis (McGraw-Hill Book Co., Ine., New York, 1957), Thap. 9.

6. J. M. B1att, "Practical Polnts Concerning the Solution of the Schrodinger Equation," J. Comput. Phys. 1, 382-396 (1967). 